\title{
COMPREHENSIVE GERIATRIC CARE VERSUS STANDARD CARE FOR ELDERLY REFERRED TO A REHABILITATION UNIT - A RANDOMIZED CONTROLLED TRIAL
}

\author{
D. Zintchouk ${ }^{1}$ T. Lauritzen' ${ }^{2}$ E.M. Damsgaard ${ }^{1}$
}

\begin{abstract}
Objective: To investigate the effect of comprehensive geriatric care (CGC) in elderly referred to a rehabilitation unit. This article describes the considerations behind the study. Design: Participants were randomized to either CGC or standard care. Setting: Participants were recruited from two community care rehabilitation units in Aarhus Municipality, Denmark, in the period between 2012 and 2015. Participants: Inclusion: Elderly patients aged 65 and older admitted from home or hospital. Exclusion: Persons receiving palliative care or assessed by a geriatrician during the past month. Intervention: Medical history, physical examination, blood tests, medication adjustment and follow-up by a geriatrician. The control group received standard care with the general practitioners (GPs) as back-up. Outcomes: Primary outcome: Hospital contacts drawn from national registers. Secondary outcomes: GPs contacts, institutionalization, medication status and mortality collected from national registers, activities of daily living (ADL), physical and cognitive function and quality of life measures collected by a blinded occupational therapist. All outcomes were assessed at day 10, 30 and 90 after arrival at the rehabilitation unit. Conclusion: A new model of care for elderly referred to community rehabilitation was developed and implemented. The potential benefits of this model were compared with usual care in a community rehabilitation unit in a pragmatic randomized clinical trial. We hypothesized that the geriatrician-performed CGC in elderly referred to a rehabilitation unit will reduce the hospital contacts by $25 \%$ without increase in mortality and in contacts to GPs and home care services. We expect that this model will prevent deterioration in ADL, physical and cognitive functioning, and reduce the risk of institutionalization. If the results are positive, community rehabilitation services should be encouraged to change their routines for treatment of this population accordingly.
\end{abstract}

Key words: Randomized controlled trial, comprehensive geriatric care, hospitalization, rehabilitation, activity of daily living.

\section{Introduction}

Older people are the fastest growing sector of the population and they account for the largest increase in hospital admissions (1). More survivors with chronic diseases mean increasing numbers of overlapping comorbidities and increased risk of acute illness $(2,3)$. Admissions to hospital for older people are combined with risk of rapid decline in functional ability, cognitive impairment, and change to residential care $(4,5)$. Despite a multitude of efforts to reduce hospital attendances and admissions worldwide, the numbers are increasing year after year (6).

To give patients the best life possible and to save health care resources, we intend to evaluate the effect

1. Aarhus University Hospital, Department of Geriatrics, Aarhus C, Denmark; 2. Department of Public Health, Section of General Medical Practice, Aarhus University, Aarhus C, Denmark.

Corresponding Author: Dmitri Zintchouk, MD, Aarhus University Hospital, Department of Geriatrics, P.P. Oerumsgade 11, 8000 Aarhus C, Denmark, dmizin@ rm.dk, tel. 004526700903, fax 004578461930. of Comprehensive Geriatric Care performed by a geriatrician in a community operated rehabilitation unit.

\section{Comprehensive geriatric assessment (CGA) and comprehensive geriatric care (CGC)}

CGA is defined as a "multidimensional interdisciplinary diagnostic process focusing on a frail older person's medical, psychological and functional capability"(7). In practice the assessment is followed by an intervention and sometimes by a follow-up based on the assessment. The recently suggested concept of comprehensive geriatric care (CGC) covers the combined assessment and follow-up interventional process more precisely (8).

Several models of CGA and CGC have been proposed. The last meta-analysis from Ellis et al. (9) showed that only inpatient CGA in acute geriatric units is effective and results in an increased likelihood of a patient returning home and avoiding admission to residential care or deterioration and death. Randomized studies of post- 
Figure 1

Study flow in the Comprehensive Geriatric Care versus Standard Care for Elderly referred to a Rehabilitation Unit a Randomized Controlled Trial

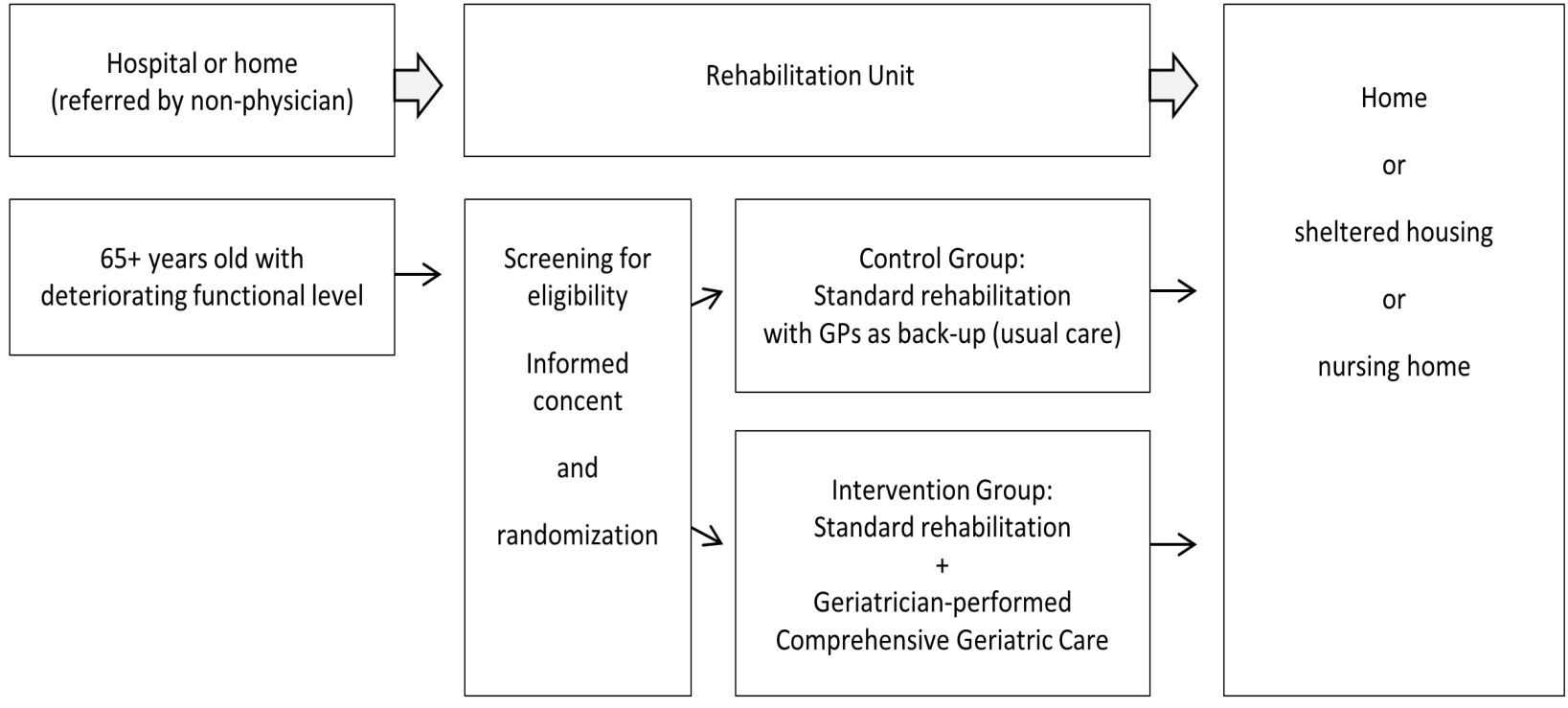

hospital discharge CGA found inconsistent benefits in functional status, acute care visits, depression, and patient satisfaction $(10,11)$. However, post-discharge intervention was associated with reduction in costs and readmission rates $(12,13)$, and CGC may be beneficial for hip fracture patients by reducing complications, mortality, readmissions, and delirium $(8,14-17)$.

A few randomized studies on different care models were published in the last five years. Senior and colleagues (18) showed that the model of restorative care services delivered within both residential care and at home by a multi-disciplinary team, included a case manager, nurse, occupational therapist and physiotherapist, tend to reduce the risk of death or permanent residential care. The absolute risk reduction for death or permanent residential care of $14.3 \%$ was not significant compared to usual care group at 24 months follow-up. Moreover, the intervention group had more frequent utilization of personal care, home help, career support, respite, day center and day activity centers than the usual care group. The same research group (19) showed that locally based care model managed by experienced nurses working with strong partnerships with family physicians reduces the risk of death and permanent residential care placement in frail older adults by $10.2 \%$ compared to usual community care coordinated by a centrally based needs assessor.

A recent Danish study shows that home-visits by a geriatrician and a specialized nurse on the first days after discharge from hospital reduce the readmission rate for acute medical patients by almost $50 \%$, compared to patients accompanied home or subsequently receiving a telephone call. Rehospitalization was reduced, but 30-day mortality did not differ significantly between groups (20).

\section{Geriatrician-performed comprehensive geriatric care in community rehabilitation settings}

Physicians alone can perform many aspects of CGA followed by intervention. Often this is not practicable given the limited time available and the workload of instituting a complex care plan (21). We have deliberately chosen to focus on the role of the geriatrician in community rehabilitation. The staff of community rehabilitation units has some expertise in care of elderly with deteriorating function. Involvement of a larger team from the geriatric department may confuse the patients and cause unnecessary expenditure.

To our knowledge, no randomized studies have evaluated the effect of geriatrician-performed CGC comprising CGA and intervention with follow-up in elderly referred to a community rehabilitation unit.

\section{Objective}

The objective of this study is to investigate the effect of the geriatrician-performed CGC compared to a control group with standard care in elderly referred to a community rehabilitation unit.

\section{Methods}

The study is a pragmatic open assessor-blinded randomized clinical trial with 90 days' follow-up. 


\section{Participants and settings}

The inclusion criteria were: 1) age 65 years or older; 2) referral to a community rehabilitation unit from home or a hospital department. The exclusion criteria were: 1) palliative care; 2 ) assessment by a geriatrician during the past one month. The participants were all residents of two community rehabilitation units, Vikaergaarden (64 rooms) and Thorsgaarden (24 rooms) in Aarhus Municipality, Denmark. For study flow, see Figure.

\section{Recruitment}

Participants were consecutively recruited from unit Vikaergaarden in the period January 17, 2012 to May 29, 2015, and from unit Thorsgaarden from October 20, 2014 to May 29, 2015. Eligible elderly and/or their relatives were contacted by the project manager or research nurse, who provided the oral and written information. Participants with cognitive impairment were also included. All had twenty-four hours to consider or discuss with relatives before the written informed consent was obtained.

During the study enrolment the following adjustments were made to accelerate the inclusion of the participants: inclusion age was lowered from 70+ to 65+ from May 14, 2012 , previous contact with a geriatrician within three months was reduced to one month from December 2, 2012. All the changes have been submitted to Clinical. Trials.gov (NCT01506219).

\section{Randomization}

The random allocation of the participants to the intervention and control groups was done by an independent external organization ("TrialPartner", Public Health and Quality Improvement, Central Denmark Region). The permuted block sizes stratified the randomization according to sex, age and place of referral. The randomization took place within three days after the participants' arrival to the rehabilitation unit. In the intervention group the geriatrician informed participants and relatives about the allocation and gave the personal contact information card to participants or relatives.

\section{Blinding}

Owing to the nature of this study, it was impossible to blind participants and their relatives to the allocation group. The project manager screened the patients for eligibility, collected data on age, gender, place of referral and comorbidity before randomization, and conducted the intervention. The project manager had no contact with the control participants after randomization. The project manager was blinded to the study outcomes, which were collected from the registers or by the blinded research occupational therapist. Rehabilitation units' staffs, particularly physiotherapists, were not blinded.

\section{Standard care in the rehabilitation unit}

The patients were referred for rehabilitation either from hospital or home by the hospital personnel or by the home care staff. Rehabilitation services are not free of charge, and a moderate fee for the stay is paid by the patients themselves. The typical standard rehabilitation and care program lasts five weeks. The interdisciplinary approach is based on the patient's whole situation, capability and wishes/needs. On the first day of rehabilitation, the patient's functional status is observed by the rehabilitation unit's physiotherapists and occupational therapists, and a nutritional screening is performed by the rehabilitation unit's nutritionist. The team members discuss the patient's discharge destination and necessary arrangements with the patient and his/her relatives at the mid-term meeting and before discharge from the rehabilitation unit. Municipality nurse participates in these meeting personally or by telephone. Destination after discharge is based upon the patient's motivation, functional and medical status.

The patient's GPs visit the patients during the stay if required or occasionally by own initiative depending on practice routine and geographical distance. GPs mostly visit frail and high-risk elderly patients especially if recently hospitalized. Acute medical aid is called for in case of illness after 4.p.m. and on weekends and public holidays.

\section{Care in the intervention group}

Participants randomized to the intervention group underwent the geriatrician-performed CGC during the rehabilitation stay. The intervention was performed by a physician specialized in geriatric medicine. The primary assessments lasted about an hour and included review of diagnoses, organ functional status, medication, and life expectancy evaluation. Individual disease management and coping was provided using the holistic approach during the face-to-face counselling, where the actual problems, expectations and aims were defined in dialogue with the patient and/or relatives. Afterwards, targeted problem solving with focus on the potentially reversible causes of functional deterioration was established. Finally, medication adjustment was carried out with particular attention to drugs which may lead to iatrogenic functional deterioration, delirium, falls, and malnutrition. A simple tool like the STOPP (Screening Tool of Older Person's Prescriptions) and START (Screening Tool to Alert doctors to Right Treatment) criteria have been used as an evidence-based approach to reduce inappropriate prescribing and to encourage appropriate prescribing in the older adult $(22,23)$. When no evidence base existed for drug use, the approach was 
Table 1

Patient treatment in the Comprehensive Geriatric Care versus Standard Care for Elderly referred to a Rehabilitation Unit - a Randomized Controlled Trial

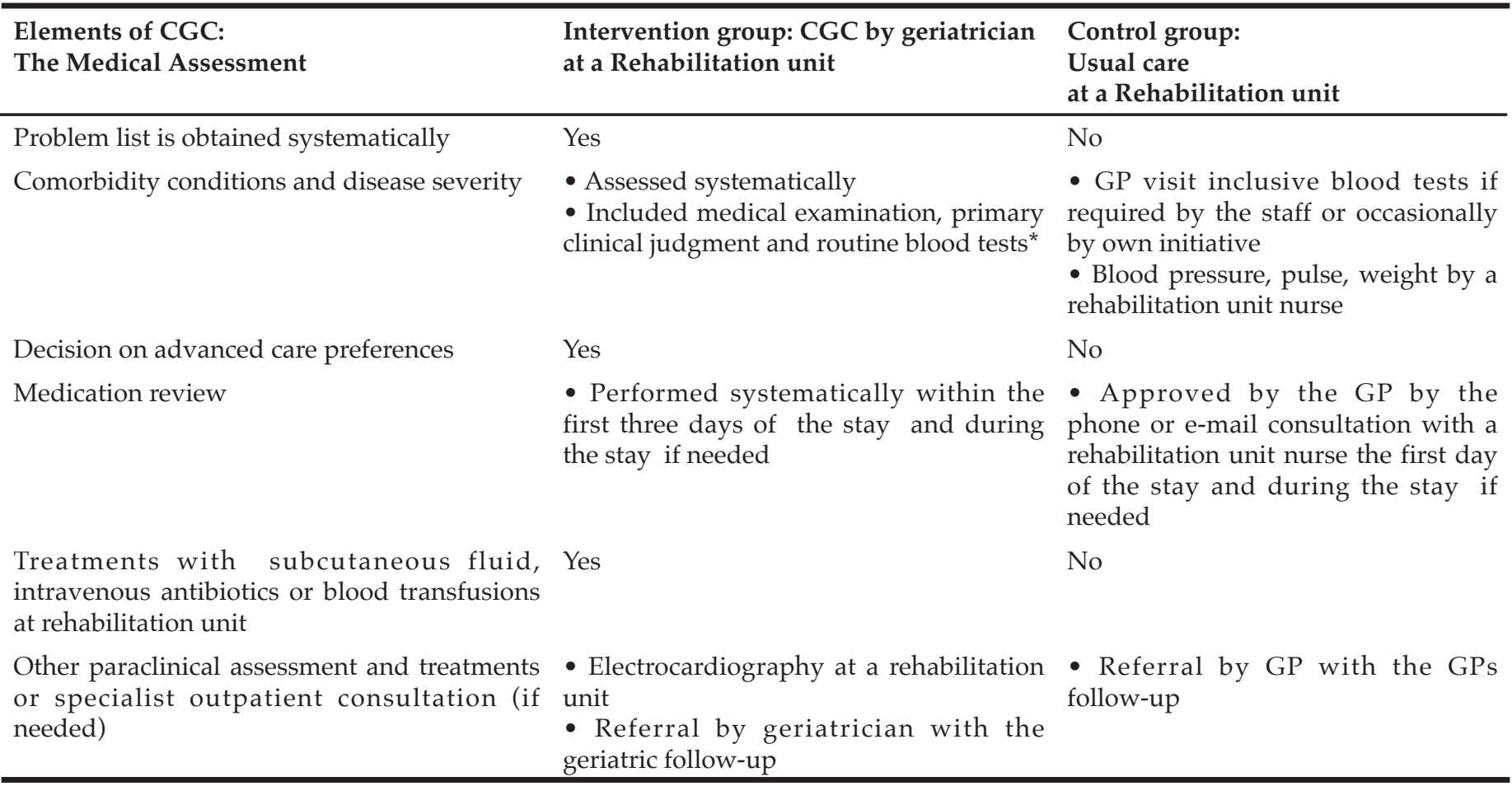

* Hemoglobin, Leucocytes, C-reactive protein, P-albumin, P-Potassium, P-Sodium, glomerular filtration rate.

based on clinical judgment only, and the balance of risks and benefits of the drug for the individual was presented to the participants and/or relatives. In collaboration with rehabilitation unit's staff the geriatrician followed the participants with regard to any change in symptoms, signs, or relevant laboratory and diagnostic test results that might indicate a restart of a specific medication, which had been discontinued.

The geriatrician was present at the rehabilitation unit for about four days a week, and could be contacted on telephone for any reason by participants, their relatives or the unit's staff on weekdays from 8 a.m. to 3 p.m. In acute situations the geriatrician could also be contacted. The follow-up period by the geriatrician at the rehabilitation units was individualized (generally four weeks). The geriatrician sent the discharge summary for each intervention group participant to the GP. The geriatrician also provided education and support to the staff of the rehabilitation units and informed and advised the GPs and primary care services if needed. After discharge from the rehabilitation units GP are responsible for treatment.

See Table 1 for patient treatment in the intervention versus control group.

\section{Outcomes}

\section{Baseline data}

Baseline characteristics were registered by the project manager from medical records and/or interview, comprising age, gender, place of referral (own home or hospital), marital status, residential status, diagnoses, comorbidity, and list of medications. The functional tests and quality of life at baseline (day 3) were done by the research occupational therapist after randomization.

\section{Primary outcome}

Primary outcome was total number of hospital contacts within 90 days after admission to the rehabilitation units.

\section{Secondary outcomes}

Secondary outcomes included all hospital and GPs contacts and number of participants with the hospital and GPs contacts, number of days spent in hospital, use of homecare services, transfer to nursing homes or sheltered housing, changes in medication status and number of deaths within 90 days. Moreover participant's ADL, cognitive and physical functioning, and quality of life were assessed at day 3, 10, 30 and 90 after admission 
Table 2

Outcomes in the Comprehensive Geriatric Care versus Standard Care for Elderly referred to a Rehabilitation Unit a Randomized Controlled Trial

\begin{tabular}{|c|c|c|c|c|c|c|}
\hline \multicolumn{2}{|l|}{$\begin{array}{l}{ }^{*} \text { Drawn from The National Patient Registry } \\
\text { - Recorded through the Community Care Record } \\
\text { by research nurse } \\
\text { o Obtained by blinded research therapist }\end{array}$} & $\begin{array}{l}\text { Before } \\
\text { arrival to a } \\
\text { Rehabilitation } \\
\text { Unit (day 0) }\end{array}$ & Day 1-3 & Day 10 & Day 30 & Day 90 \\
\hline Primary outcome & $\begin{array}{l}\text { Total number of hospitalization and Emergency } \\
\text { Department visits }\end{array}$ & & & & & * \\
\hline Secondary outcomes & $\begin{array}{l}\text { Planned and unplanned hospitalization, Emergency } \\
\text { Department visits, outpatient clinic visits } \\
\text { Number of days spent in hospital } \\
\text { Phone contacts to/by GP } \\
\text { Visits to/by GP } \\
\text { Home visits district nurses availability } \\
\text { Personal social services extent } \\
\text { CAM } \\
\text { MMSE } \\
\text { The } 30 \text {-second chair stand test } \\
\text { Modified Barthel-100 Index } \\
\text { Depression List } \\
\text { Residential status } \\
\text { Mortality } \\
\text { Medication status }\end{array}$ & $\bullet$ & $\begin{array}{l}0 \\
0 \\
0 \\
0 \\
0\end{array}$ & $\begin{array}{l}0 \\
0 \\
0 \\
0 \\
0\end{array}$ & $\begin{array}{l}\mathrm{O} \\
\mathrm{O} \\
\mathrm{O} \\
\mathrm{O} \\
\mathrm{O} \\
\text { * }\end{array}$ & $\begin{array}{l}\text { * } \\
\text { * } \\
\text { * } \\
\text { • } \\
\text {. } \\
0 \\
0 \\
0 \\
0 \\
0 \\
\text { * } \\
\text { * }\end{array}$ \\
\hline
\end{tabular}

to the rehabilitation units. Trial outcome follow-up was completed August, 27. 2015.

\section{Measurements}

1) Mini-mental state (MMSE) (24). MMSE is a 10-minute bedside measure of impaired thinking. The items of the MMSE include tests of orientation, registration, recall, calculation and attention, naming, repetition, comprehension, reading, writing and drawing (25).

2) The Confusion Assessment Method (CAM) (26).

CAM is a standardized evidence-based tool that enables non-psychiatrically trained clinicians to identify and recognize delirium quickly and accurately in both clinical and research settings. The CAM includes four features found to be most effective in distinguishing delirium from other types of cognitive impairment.

3) Modified Barthel-100 Index (MBI) (27).

MBI is a 10 -item instrument that provides a score of basic daily activities (feeding, bathing, grooming, dressing, bowels, bladder, toilet use, transfer, mobility, and stair climbing). The scores range from $0-100$, with a higher score indicating greater independence.

4) The 30-second chair stand test (28).

The 30-second chair stand test measures body strength, by determining the number of times the participant can stand up fully and sit down in 30 seconds, with the arms crossed over the chest. We have used the modified version of this test, where use of armrest is allowed.

5) Depression List (DL) (29).

DL is a fifteen-item questionnaire, designed to assess quality of life in frail nursing home residents. DL addresses emotional well-being, social relationships, life satisfaction, comfort, functional competence, and autonomy. The scale ranges from 0 (best quality of life) to score 30 (poorest quality of life).

6) Charlson Comorbidity Index (CCI) (30) is used to categorize comorbidity in three levels:

$0=$ low, $1-2=$ moderate, and 3 or more $=$ high .

All the functional measurements, except for the modified version of "The 30-second chair stand test", are validated for use in an elderly population. All questionnaires were performed as structured interviews. Trial outcome follow-up was completed August, 27. 2015.

\section{Data collection}

Data on hospital contacts and GPs contacts and mortality were collected from The National Patient Registry, The National Health Insurance Service Register and Danish Civil Registration System via Researcher Service, Statens Serum Institut, Danish Ministry of Health. Data on causes on hospital contacts were collected by the primary investigator from the Electronic Patient Record. Data on district nurses availability, extent of personal social services, walking aids and residential status were recorded through the Aarhus Community Care Record.

The actual medication usage and the number of Defined Daily Doses (DDD) within the different The Anatomical Therapeutic Chemical (ATC) Classification System were clarified and recorded under the personal medication review and through the Electronic Patient Record and the Aarhus Community Care Record by the primary investigator and the research nurse.

The functional tests and evaluation of the healthrelated quality of life during the follow-up period were performed by the research occupational therapist.

For data collection details, see Table 2. 


\section{Ethics}

Written informed consent was obtained from the participants by the project manager or research nurse within two days of arrival at the rehabilitation unit. Under the consent procedure the project manager assessed the elderly's cognitive capacities. Cognitive impairment was defined by: (1) MMSE score of $<25$; (2) CAM indicating delirium; or (3) a clinical cognitive evaluation undertaken by the project manager. Patients who were not cognitively impaired gave their written informed consent. Consent of cognitively impaired patients was given by a relative.

The project manager informed the participant's GPs by letter about the study participation without information about the allocation. In the intervention group the GPs were shortly informed by the geriatrician about the treatment plan per mail in the Electronic Patient Record.

The CGC contained all known and commonly used and approved testing methods. All data are treated in confidence and participants are assured anonymity. The study is approved by the Danish Data Protection Agency, journal no. 2012-58-006, and the Ethical Committee of Central Denmark Region, journal no. M-20110262.

An interim-analysis was performed on the mortality when $50 \%$ of participants have been randomized and have completed the 90 days' follow-up. The interimanalysis was performed by an independent statistician, blinded for the treatment allocation. Results were evaluated by an independent researcher in order to stop the study prematurely if significant mortality differences were found.

\section{Sample size and data analysis}

\section{Power calculation}

For power calculation we used data on hospital contacts from The National Patient Registry in persons receiving rehabilitation at the rehabilitation unit Vikaergaarden from 1 April 2009 to 31 March 2010. There were 153 hospital contacts among 550 65+ year old persons within three months after the admission at rehabilitation. An analysis of hospital contacts over 30 days in 68 participants in a pilot project showed $33 \%$ fewer hospital contacts in the intervention group (number of persons with hospital contacts $=7$, total number of contacts=12) compared to the control group (number of persons with hospital contacts $=7$, total number of contacts $=19$ ).

For the sample size calculation we expected a $25 \%$ reduction of the hospital contacts, which we regarded as a clinically relevant change. Estimated dropout was set to $20 \%$ in both groups, as mortality was expected to be high. To obtain $80 \%$ statistical power and a significance level at 0.05 we had to recruit 370 patients.

\section{Data analysis}

All data are being entered in a database (Access 2010) by the research nurse. The statistical analyses will be conducted based on a predefined statistical protocol using STATA (version 13, STATA Corporation, Texas). Both descriptive and analytic analysis will be performed. Descriptive data will be calculated in percent, while median, average and minimum and maximum will be used for continuous variables. Continuous variables will be analyzed for normal distribution with the Kolmogorov-Smirnov test. The principle of repeated measurements will be used to analyze continuous variables. Variables with dichotomous outcomes will be analyzed using the logistic regression. Non-normally distributed data will be analyzed with the MannWhitney U test/Wilcoxon matched-pair's test. Mortality will be analyzed with Kaplan-Meier analysis. Survival analysis will be performed with Cox Regression model adjusting for the sex, age, comorbidity and place of referral. In order to ensure the statistical robustness of the intervention outcomes, two different longitudinal imputation methods (last value carried forward and worst value imputation) will be used in case of missing values on sensitive analysis. There will be a bilateral significance level of $5 \%$ for evaluation of statistical significance in the primary and secondary endpoints. Intention-to-treat analysis will be performed.

\section{Discussion}

To our knowledge this is the first randomized controlled study to evaluate the effect of the CGC performed by a geriatrician in elderly citizens referred to community rehabilitation. In a systematic review the authors found that no particular model of geriatric care in community rehabilitation facilities could be recommended (31). In spite of multiple recent advances in providing rehabilitation in community settings, organization of these services, particularly the role of the geriatrician, remains poorly addressed.

\section{Strengths and limitations of the study}

\section{Design}

The RCT design was chosen to investigate the broad population of elderly with functional loss and multimorbidity, often excluded from RCTs (32-34). This must be considered as a strength. However, it has a price because the heterogeneity of the study population requires a much greater number of participants to demonstrate a possible significant difference. 


\section{Study population}

The strength of the study population was the broad inclusion criteria, which insured enrolment of participants with a wide range of medical conditions.

We also decided to include elderly with dementia or confusion on arrival at the rehabilitation unit. We expected these persons to benefit most from the geriatrician-performed CGC.

On the other hand the recruitment was expected to be challenged due to difficulties in obtaining written informed consent. In order to detect possible selection bias among participants information about age, gender, place of referral and comorbidity (CCI) was obtained for participants as well as non-participants.

\section{Intervention}

The intervention was individualized and holistic based on a dialogue with the patient and/or relatives setting realistic common aims and expectation for treatment. This pragmatic clinical approach attempts to maximize external validity (35). The individual needs of the elderly are complicated by medical, functional, psychological, and social problems (36).This may lead to an atypical clinical presentation requiring flexibility and variation of the treatment.

Yet, the intervention was as systematic as possible in order to be reproducible. However, the medication adjustments by the geriatrician were not standardized. We were not able to use the STOPP-START tool strictly due to a systematic lack of the patient dimension. Medicine adjustments in elderly may conflict with established guidelines not addressing the care of people with multiple conditions (37). Such discrepancies may confuse the participant, the home career or the GP and result in readministration of discontinued drugs.

A specialist physician in geriatric medicine performed the intervention. This has strengths: the rehabilitation units' staff could easily contact the geriatrician, who was physically available at an office in the rehabilitation units. In the majority of cases the primary investigator/project manager was also the geriatrician who conducted the intervention. It makes continuity possible and optimizes communication with the patients, their relatives and the staff of the rehabilitation units. It may promote the compliance and be more cost effective. On the other hand when the intervention depends on one physician the results are less generalizable and should be confirmed by further studies.

A stronger cooperation was established between the geriatric department and the rehabilitation units, likewise educational courses on common geriatric problems were carried out for the staff during the study period. Both the intervention and the control group were treated by the same personnel, which may have a positive spillover effect reducing a possible difference between the groups.

\section{Blinding}

A strength of our study was the systematical efforts tried to minimize information bias. The geriatrician was blinded to the primary endpoint data that were drawn from The National Patient Registry via Researcher Service. The dataset was generated by the Registry's staff blinded to the patient allocation.

It was a weakness that it was impossible to blind the participants and their relatives or the geriatrician and the rehabilitation units' staff to the allocation group. The research nurse was not blinded to patient allocation for practical reasons. The research occupational therapist was blinded to treatment allocation, but it could not be ruled out that the participants may have mentioned their allocation during the assessment. Thus, the performancebased measure of physical and cognitive functioning could be biased.

\section{Outcome measures}

A strength of this study was the use of functional measurements and questionnaires well validated for elderly. The modified version of the " 30 -second chair stand test", in which the use of armrest is allowed, was the only test not validated. However, it is suited for our study population, as the majority of the participants were not able to perform the original version of the test.

\section{Conclusion}

A new model of care for elderly referred to community rehabilitation was developed and implemented. The potential benefits of this model were compared with usual care in a community rehabilitation unit in a pragmatic randomized clinical trial. This pragmatic approach closely mimics the true clinical situation. We hypothesize that the geriatrician-performed CGC in elderly referred to a rehabilitation unit will reduce the hospital contacts by $25 \%$. This should be done without increasing mortality, GP contacts or home care services. We expect this model to prevent deterioration in ADL, physical and cognitive functioning, and to reduce the risk of institutionalization. Data collection was recently completed. The results may soon be published.

Acknowledgements: This trial is funded by Geriatric Department Aarhus University Hospital and received donations from the Health Insurance Fund (Helsefonden) and Public Health in the Central Region Fund (Folkesundhed i Midten).

Authors' contributions: DZ in collaboration with EMD and TL designed the study. DZ carried out the interventions. DZ drafted the manuscript. All authors revised the manuscript critically and have given their final approval of this version to be published.

Conflict of Interest: There is no conflict of interest to declare. DZ is a specialist in geriatrics and a member of network of Danish physicians and medical students "Physicians without sponsor". 


\section{References}

1. OECD. "Elderly population"(indicator). DOI: http://dx.doi. org/10.1787/8d805ea1-en (Accessed on 02 July 2016). 2014 Jun 25.

2. Barnett K, Mercer SW, Norbury M, Watt G, Wyke S, Guthrie B. Epidemiology of multimorbidity and implications for health care, research, and medical education: a cross-sectional study. Lancet 2012 Jul 7;380(9836):37-43.

3. Roberts DC, McKay MP, Shaffer A. Increasing rates of emergency department visits for elderly patients in the United States, 1993 to 2003. Ann Emerg Med 2008 Jun;51(6):769-774.

4. Covinsky KE, Palmer RM, Fortinsky RH, Counsell SR, Stewart AL, Kresevic $\mathrm{D}$, et al. Loss of independence in activities of daily living in older adults hospitalized with medical illnesses: increased vulnerability with age. J Am Geriatr Soc 2003 Apr;51(4):451-458.

5. Mudge AM, O'Rourke P, Denaro CP. Timing and risk factors for functional changes associated with medical hospitalization in older patients. J Gerontol A Biol Sci Med Sci 2010 Aug;65(8):866-872.

6. van Walraven C, Bennett C, Jennings A, Austin PC, Forster AJ. Proportion of hospital readmissions deemed avoidable: a systematic review. CMAJ 2011 Apr 19;183(7):E391-402.

7. Rubenstein LZ, Stuck AE, Siu AL, Wieland D. Impacts of geriatric evaluation and management programs on defined outcomes: overview of the evidence. J Am Geriatr Soc 1991 Sep;39(9 Pt 2):8S-16S; discussion 17S-18S.

8. Prestmo A, Hagen G, Sletvold O, Helbostad JL, Thingstad P, Taraldsen K, et al. Comprehensive geriatric care for patients with hip fractures: a prospective, randomised, controlled trial. Lancet 2015 Apr 25;385(9978):1623-1633.

9. Ellis G, Whitehead MA, O'Neill D, Langhorne P, Robinson D. Comprehensive geriatric assessment for older adults admitted to hospital. Cochrane Database Syst Rev 2011 Jul 6;(7):CD006211. doi(7):CD006211.

10. Siu AL, Kravitz RL, Keeler E, Hemmerling K, Kington R, Davis JW, et al. Postdischarge geriatric assessment of hospitalized frail elderly patients. Arch Intern Med 1996 Jan 8;156(1):76-81.

11. Naylor MD, Brooten D, Campbell R, Jacobsen BS, Mezey MD, Pauly MV, et al. Comprehensive discharge planning and home follow-up of hospitalized elders: a randomized clinical trial. JAMA 1999 Feb 17;281(7):613-620.

12. Naylor MD, Aiken LH, Kurtzman ET, Olds DM, Hirschman KB. The care span: The importance of transitional care in achieving health reform. Health Aff (Millwood) 2011 Apr;30(4):746-754.

13. Hesselink G, Schoonhoven L, Barach P, Spijker A, Gademan P, Kalkman C, et al. Improving patient handovers from hospital to primary care: a systematic review. Ann Intern Med 2012 Sep 18;157(6):417-428.

14. Bjorkelund KB, Hommel A, Thorngren KG, Gustafson L, Larsson S, Lundberg D. Reducing delirium in elderly patients with hip fracture: a multi-factorial intervention study. Acta Anaesthesiol Scand 2010 Jul;54(6):678-688.

15. Gregersen M, Zintchouk D, Borris LC, Damsgaard EM. A geriatric multidisciplinary and tailor-made hospital-at-home method in nursing home residents with hip fracture. Geriatr Orthop Surg Rehabil 2011 Jul;2(4):148-154.

16. Fisher AA, Davis MW, Rubenach SE, Sivakumaran S, Smith PN, Budge MM. Outcomes for older patients with hip fractures: the impact of orthopedic and geriatric medicine cocare. J Orthop Trauma 2006 Mar;20(3):172-8; discussion 179-80.

17. Marcantonio ER, Flacker JM, Wright RJ, Resnick NM. Reducing delirium after hip fracture: a randomized trial. J Am Geriatr Soc 2001 May;49(5):516-522.

18. Senior HE, Parsons M, Kerse N, Chen MH, Jacobs S, Hoorn SV, Anderson CS. Promoting independence in frail older people: a randomised controlled trial of a restorative care service in New Zealand. Age Ageing, 2014, 43, 3, 418-424.
19. Parsons M, Senior H, Kerse N, Chen MH, Jacobs S, Vanderhoorn S, et al. Should care managers for older adults be located in primary care? A randomized controlled trial. J Am Geriatr Soc 2012 Jan;60(1):86-92.

20. Pedersen LH, Gregersen M, Barat I, Damsgaard EM. Early geriatric follow-up after discharge reduces readmissions - A quasi-randomised controlled trial. European Geriatric Medicine. In Press.

21. Moore AA, Siu AL. Screening for common problems in ambulatory elderly: clinical confirmation of a screening instrument. Am J Med 1996 Apr;100(4):438-443.

22. Gallagher P, O'Mahony D. STOPP (Screening Tool of Older Persons' potentially inappropriate Prescriptions): application to acutely ill elderly patients and comparison with Beers' criteria. Age Ageing 2008 Nov;37(6):673679 .

23. Gallagher P, Ryan C, Byrne S, Kennedy J, O'Mahony D. STOPP (Screening Tool of Older Person's Prescriptions) and START (Screening Tool to Alert doctors to Right Treatment). Consensus validation. Int J Clin Pharmacol Ther 2008 Feb;46(2):72-83.

24. Folstein MF, Folstein SE, McHugh PR. "Mini-mental state". A practical method for grading the cognitive state of patients for the clinician. J Psychiatr Res 1975 Nov;12(3):189-198

25. Cockrell JR, Folstein MF. Mini-Mental State Examination (MMSE). Psychopharmacol Bull 1988;24(4):689-692.

26. Inouye SK, van Dyck CH, Alessi CA, Balkin S, Siegal AP, Horwitz RI Clarifying confusion: the confusion assessment method. A new method for detection of delirium. Ann Intern Med 1990 Dec 15;113(12):941-948.

27. Shah S, Vanclay F, Cooper B. Improving the sensitivity of the Barthel Index for stroke rehabilitation. J Clin Epidemiol 1989;42(8):703-709.

28. Jones CJ, Rikli RE, Beam WC. A 30-s chair-stand test as a measure of lower body strength in community-residing older adults. Res Q Exerc Sport 1999 Jun;70(2):113-119.

29. Gerritsen DL, Steverink N, Ooms ME, de Vet HC, Ribbe MW. Measurement of overall quality of life in nursing homes through self-report: the role of cognitive impairment. Qual Life Res 2007 Aug;16(6):1029-1037.

30. Charlson ME, Pompei P, Ales KL, MacKenzie CR. A new method of classifying prognostic comorbidity in longitudinal studies: Development and validation. J Chronic Dis 1987; 40 (5): 373-83.

31. Parker G, Bhakta P, Katbamna S, Lovett C, Paisley S, Parker S, et al. Best place of care for older people after acute and during subacute illness: a systematic review. J Health Serv Res Policy 2000 Jul;5(3):176-189.

32. Bourgeois FT, Olson KL, Tse T, Ioannidis JP, Mandl KD. Prevalence and Characteristics of Interventional Trials Conducted Exclusively in Elderly Persons: A Cross-Sectional Analysis of Registered Clinical Trials. PLoS One 2016 May 19;11(5):e0155948.

33. Konrat C, Boutron I, Trinquart L, Auleley GR, Ricordeau P, Ravaud P. Underrepresentation of elderly people in randomised controlled trials. The example of trials of 4 widely prescribed drugs. PLoS One 2012;7(3):e33559.

34. Van Spall HG, Toren A, Kiss A, Fowler RA. Eligibility criteria of randomized controlled trials published in high-impact general medical journals: a systematic sampling review. JAMA 2007 Mar 21;297(11):1233-1240.

35. Zwarenstein M, Treweek S, Gagnier JJ, Altman DG, Tunis S, Haynes B, et al. Improving the reporting of pragmatic trials: an extension of the CONSORT statement. BMJ 2008 Nov 11;337:a2390.

36. Gray L. Geriatric consultation: is there a future? Age Ageing 2007 Jan;36(1):12.

37. Guthrie B, Payne K, Alderson P, McMurdo ME, Mercer SW. Adapting clinical guidelines to take account of multimorbidity. BMJ 2012 Oct 4;345:e6341. 